\title{
Askey-Wilson integral and its generalizations
}

Paweł J Szabłowski*

${ }^{*}$ Correspondence:

pawel.szablowski@gmail.com

Department of Mathematics and Information Sciences, Warsaw

University of Technology,

ul. Koszykowa 75, Warsaw 00-662,

Poland

\begin{abstract}
We expand the Askey-Wilson (AW) density in a series of products of continuous q-Hermite polynomials times the density that makes these polynomials orthogonal. As a by-product we obtain the value of the AW integral as well as the values of integrals of $q$-Hermite polynomial times the AW density ( $q$-Hermite moments of AW density). Our approach uses nice, old formulae of Carlitz and is general enough to venture a generalization. We prove that it is possible and pave the way how to do it. As a result, we obtain a system of recurrences that if solved successfully gives a sequence of generalized AW densities with more and more parameters.
\end{abstract}

MSC: 33D45; 05A30; 05E05

Keywords: Askey-Wilson integral; Askey-Wilson polynomials; q-Hermite polynomials; expansion of ratio of densities; symmetric functions

\section{Introduction and preliminaries}

\subsection{Introduction}

We consider a sequence of nonnegative, integrable functions $g_{n}:[-1,1] \mapsto \mathbb{R}^{+}$defined by the formula

$$
g_{n}\left(x \mid \mathbf{a}^{(n)}, q\right)=f_{h}(x \mid q) \prod_{j=1}^{n} \varphi_{h}\left(x \mid a_{j}, q\right),
$$

where $\mathbf{a}^{(n)}=\left(a_{1}, \ldots, a_{n}\right)$, functions $f_{h}$ and $\varphi_{h}$ defined by (1.16) and (1.14) denote respectively the density of measure that makes the so-called continuous $q$-Hermite polynomials orthogonal and the generating function of these polynomials calculated at points $a_{j}$, $j=1, \ldots, n$. Naturally functions $g_{n}$ are symmetric with respect to vectors $\mathbf{a}^{(n)}$.

Our elementary but crucial for this paper observation is that examples of such functions are proportional to the densities of measures that make orthogonal respectively the socalled continuous $q$-Hermite ( $q$-Hermite, $n=0$ [1, Eq. (14.26.2)]), big $q$-Hermite (bqH, $n=1$ [1, Eq. (14.18.2)]), Al-Salam-Chihara (ASC, $n=2$ [1, Eq. (14.8.2)]), continuous dual Hahn (C2H, $n=3$ [1, Eq. (14.3.2)]), Askey-Wilson (AW, $n=4$ [1, Eq. (14.1.2)]) polynomials. This observation makes functions $g_{n}$ important and, what is more exciting, allows possible generalization of both AW integral and AW polynomials, i.e., go beyond $n=4$.

Similar observations were made in fact in [2] when commenting on formula (10.11.19). Hence one can say that we are developing a certain idea of [2].

Let us notice that this is a second attempt to generalize AW polynomials. The other one was made in [3] by generalizing certain properties of generating functions of $q$-Hermite, bqH, ASC, $\mathrm{C} 2 \mathrm{H}$ and $\mathrm{AW}$ polynomials.

\section{Springer}

C2014 Szabłowski; licensee Springer. This is an Open Access article distributed under the terms of the Creative Commons Attribution License (http://creativecommons.org/licenses/by/2.0), which permits unrestricted use, distribution, and reproduction in any medium, provided the original work is properly cited. 
On the other hand, by the observation that these functions are symmetric in variables $\mathbf{a}^{(n)}$ we enter the fascinating world of symmetric functions.

The paper is organized as follows. Next Section 1.2 presents notation that will be used and basic families of orthogonal polynomials that will appear in the sequel. We also present here important properties of these polynomials.

Section 2 is devoted to expanding functions $g_{n}$ in the series of the form

$$
g_{n}\left(x \mid \mathbf{a}^{(n)}, q\right)=A_{n}\left(\mathbf{a}^{(n)}, q\right) f_{h}(x \mid q) \sum_{j \geq 0} \frac{T_{j}^{(n)}\left(\mathbf{a}^{(n)}, q\right)}{(q)_{j}} h_{j}(x \mid q),
$$

where $\left\{h_{n}\right\}$ denote $q$-Hermite polynomials, $\left\{T_{j}^{(n)}\right\}$ are the sequences of certain symmetric functions and finally $\left\{A_{n}\right\}$ are the values of the integrals

$$
\int_{-1}^{1} g_{n}\left(x \mid \mathbf{a}^{(n)}, q\right) d x
$$

and symbol $(q)_{j}$ is explained at the beginning of the next subsection.

We do this effectively for $n=0, \ldots, 4$, obtaining known results in a new way. In Section 3 we show that sequences defined above do exist, and we present the way how to obtain them recursively. We are unable, however, to present nice compact forms of these sequences resembling those obtained for $n \leq 4$, thus posing several open questions (see Section 3.2) and leaving the field to younger and more talented researchers.

The partially legible, although not very compact, form was obtained for $\int_{-1}^{1} g_{5}\left(x \mid \mathbf{a}^{(5)}, q\right) d x$ (see (3.4)).

For $q=0$, the case important for the rapidly developing so-called free probability, we give a simple, compact form for $\int_{-1}^{1} g_{5}\left(x \mid \mathbf{a}^{(5)}, 0\right) d x$ (see Theorem 2(ii)) paving the way to conjecture the compact form of (3.4).

Tedious proofs are shifted to Section 4.

\subsection{Preliminaries}

$q$ is a parameter. We will assume that $-1<q \leq 1$ unless otherwise stated. Let us define $[0]_{q}=0,[n]_{q}=1+q+\cdots+q^{n-1},[n]_{q} !=\prod_{j=1}^{n}[j]_{q}$, with $[0]_{q} !=1$ and

$$
\left[\begin{array}{l}
n \\
k
\end{array}\right]_{q}= \begin{cases}\frac{[n]_{q} !}{[n-k]_{q} ![k] q !}, & n \geq k \geq 0 \\
0, & \text { otherwise. }\end{cases}
$$

We will use the so-called $q$-Pochhammer symbol for $n \geq 1$,

$$
\begin{aligned}
& (a ; q)_{n}=\prod_{j=0}^{n-1}\left(1-a q^{j}\right), \\
& \left(a_{1}, a_{2}, \ldots, a_{k} ; q\right)_{n}=\prod_{j=1}^{n}\left(a_{j} ; q\right)_{n},
\end{aligned}
$$

with $(a ; q)_{0}=1$.

Often $(a ; q)_{n}$ as well as $\left(a_{1}, a_{2}, \ldots, a_{k} ; q\right)_{n}$ will be abbreviated to $(a)_{n}$ and $\left(a_{1}, a_{2}, \ldots, a_{k}\right)_{n}$ if that will not cause misunderstanding. 
It is easy to notice that $(q)_{n}=(1-q)^{n}[n]_{q}$ ! and that

$$
\left[\begin{array}{l}
n \\
k
\end{array}\right]_{q}= \begin{cases}\frac{(q)_{n}}{(q)_{n-k}(q)_{k}}, & n \geq k \geq 0 \\
0, & \text { otherwise }\end{cases}
$$

The case $q=1$ will be considered only when it might make sense and will be understood as the limit $q \rightarrow 1^{-}$.

Remark 1 Notice that $[n]_{1}=n,[n]_{1} !=n !,\left[\begin{array}{l}n \\ k\end{array}\right]_{1}=\left(\begin{array}{l}n \\ k\end{array}\right),(a ; 1)_{n}=(1-a)^{n}$ and $[n]_{0}= \begin{cases}1 & \text { if } n \geq 1, \\ 0 & \text { if } n=0 \text {, }\end{cases}$ $[n]_{0} !=1,\left[\begin{array}{l}n \\ k\end{array}\right]_{0}=1$, for $0 \leq k \leq n,(a ; 0)_{n}= \begin{cases}1 & \text { if } n=0, \\ 1-a & \text { if } n \geq 1\end{cases}$

We will need the following sets of polynomials.

The Rogers-Szegö polynomials that are defined by the equality

$$
w_{n}(x \mid q)=\sum_{k=0}^{n}\left[\begin{array}{l}
n \\
k
\end{array}\right]_{q} x^{k}
$$

for $n \geq 0$ and $w_{-1}(x \mid q)=0$. They will play an auxiliary role in the sequel.

In particular one shows (see, e.g., [4]) that the polynomials defined by

$$
h_{n}(x \mid q)=e^{i n \theta} w_{n}\left(e^{-2 i \theta} \mid q\right) \text {, }
$$

where $x=\cos \theta$, satisfy the following 3 -term recurrence:

$$
h_{n+1}(x \mid q)=2 x h_{n}(x \mid q)-\left(1-q^{n}\right) h_{n-1}(x \mid q),
$$

with $h_{-1}(x \mid q)=0, h_{0}(x \mid q)=1$.

These polynomials are the so-called continuous $q$-Hermite polynomials. A lot is known about their properties. For good reference, see [1, 4] or [5]. In particular we know that for $|q|<1$,

$$
\sup _{|x| \leq 1}\left|h_{n}(x \mid q)\right| \leq w_{n}(1 \mid q)
$$

Remark 2 Notice that $h_{n}(x \mid 0)$ equals the $n$th Chebyshev polynomial of the second kind. More about these polynomials, one can find in, e.g., [2]. To analyze the case $q=1$, let us consider rescaled polynomials $h_{n}$, i.e., $H_{n}(x \mid q)=h_{n}(x \sqrt{1-q} / 2 \mid q) /(1-q)^{n / 2}$. Then equation (1.4) takes a form

$$
H_{n+1}(x \mid q)=x H_{n}(x \mid q)-[n]_{q} H_{n-1}(x \mid q)
$$

which shows that $H_{n}(x \mid q)=H_{n}(x)$, where $\left\{H_{n}\right\}$ denote the so-called probabilistic Hermite polynomials, i.e., polynomials orthogonal with respect to the measure with density equal to $\exp \left(-x^{2} / 2\right) / \sqrt{2 \pi}$. This observation suggests that although the case $q=1$ lies within our interest, it requires special approach. In fact it will be solved completely in Section 3. For now we will assume that $|q|<1$. 
In the sequel the following identities discovered by Carlitz (see Exercise 12.3(b), (c) of [4]), true for $|q|,|t|<1$,

$$
\sum_{k=0}^{\infty} \frac{w_{k}(1 \mid q) t^{k}}{(q)_{k}}=\frac{1}{(t)_{\infty}^{2}}, \quad \sum_{k=0}^{\infty} \frac{w_{k}^{2}(1 \mid q) t^{k}}{(q)_{k}}=\frac{\left(t^{2}\right)_{\infty}}{(t)_{\infty}^{4}}
$$

will enable us to show absolute and uniform convergence of practically all series considered in the sequel.

We have also the so-called linearization formula [4, Eq. (13.1.25)], which can be dated back in fact to Rogers and Carlitz (see [2, Eq. (10.11.10)] with $\beta=0$ or [6] for Rogers-Szegö polynomials), as follows:

$$
h_{n}(x \mid q) h_{m}(x \mid q)=\sum_{j=0}^{\min (n, m)}\left[\begin{array}{c}
m \\
j
\end{array}\right]_{q}\left[\begin{array}{l}
n \\
j
\end{array}\right]_{q}(q)_{j} h_{n+m-2 k}(x \mid q),
$$

that will be our basic tool.

We will use the following two formulae of Carlitz presented in [7] that concern properties of Rogers-Szegö polynomials. Let us define two sets of functions

$$
\begin{aligned}
& \zeta_{n}(x \mid a, q)=\sum_{m \geq 0} \frac{a^{m}}{(q)_{m}} w_{n+m}(x \mid q), \\
& \lambda_{n, m}(x, y \mid a, q)=\sum_{k \geq 0} \frac{a^{k}}{(q)_{k}} w_{n+k}(x \mid q) w_{m+k}(y \mid q),
\end{aligned}
$$

defined for $|x|,|y| \leq 1,|a|<1$ and $n, m$ being nonnegative integers. Carlitz proved ([7, Eq. (3.2)], after correcting an obvious misprint) that

$$
\begin{aligned}
& \zeta_{n}(x \mid a, q)=\zeta_{0}(x \mid a, q) \mu_{n}(x \mid a, q), \\
& \zeta_{0}(x \mid a, q)=\frac{1}{(a, a x)_{\infty}},
\end{aligned}
$$

where functions $\mu_{n}$ are polynomials that are defined by

$$
\mu_{n}(x \mid a, q)=\sum_{j=0}^{n}\left[\begin{array}{l}
n \\
j
\end{array}\right]_{q}(a)_{j} x^{j}
$$

and that ([7, Eq. (1.4)], case $m=0$ also given in [4, Exercise 12.3(d)])

$$
\frac{\lambda_{m, n}(x, y \mid a, q)}{\lambda_{0,0}(x, y \mid a, q)}=\sum_{j=0}^{m} \sum_{k=0}^{n}\left[\begin{array}{l}
n \\
k
\end{array}\right]_{q}\left[\begin{array}{c}
m \\
j
\end{array}\right]_{q} \frac{(a x)_{j}(a y)_{k}(x y a)_{k+j}}{\left(x y a^{2}\right)_{k+j}} x^{m-j} y^{n-k},
$$

with

$$
\lambda_{0,0}(x, y \mid a, q)=\frac{\left(x y a^{2}\right)_{\infty}}{(a, a x, a y, a x y)_{\infty}} .
$$

It is elementary to prove the following two properties of the polynomials $\mu_{n}$, hence we present them without proof. 


\section{Proposition 1}

$$
\begin{aligned}
& x^{n} \mu_{n}\left(x^{-1} \mid a, q\right)=\sum_{j=0}^{n}\left[\begin{array}{l}
n \\
j
\end{array}\right]_{q}(-a)^{j} q^{\left(\begin{array}{l}
j \\
2
\end{array}\right)} w_{n-j}(x \mid q), \\
& w_{n}(x \mid q)=\sum_{k=0}^{n}\left[\begin{array}{l}
n \\
k
\end{array}\right]_{q} a^{k} x^{n-k} \mu_{n-k}\left(x^{-1} \mid a, q\right) .
\end{aligned}
$$

To perform our calculations, we will also need the following two functions.

The generating function of the $q$-Hermite polynomials that is given by the formula below (see [1, Eq. (14.26.1)]):

$$
\varphi_{h}(x \mid t, q) \stackrel{d f}{=} \sum_{j \geq 0} \frac{t^{j}}{(q)_{j}} h_{j}(x \mid q)=\frac{1}{\prod_{k=0}^{\infty} v\left(x \mid t q^{k}\right)},
$$

where $v(x \mid t)=1-2 t x+t^{2}$. Notice that $v(x \mid t) \geq 0$ for $|x| \leq 1$ and that from (1.5) it follows that series in (1.3) converges for $|t|<1$. Notice also that from (1.5) it follows that

$$
\sup _{|x| \leq 1} \varphi_{h}(x \mid t, q)=1 /(|t|)_{\infty}^{2} .
$$

The density of the measure with respect to which polynomials $h_{n}$ are orthogonal is given in, e.g., [1, Eq. (14.26.2)]. Following it we have

$$
\int_{-1}^{1} h_{n}(x \mid q) h_{m}(x \mid q) f_{h}(x \mid q) d x=(q)_{n} \delta_{n m}
$$

where $\delta_{m n}$ denotes Kronecker's delta, and

$$
f_{h}(x \mid q)=\frac{2(q)_{\infty} \sqrt{1-x^{2}}}{\pi} \prod_{k=1}^{\infty} l\left(x \mid q^{i}\right),
$$

where $l(x \mid a)=(1+a)^{2}-4 a x^{2}$. Notice that

$$
\sup _{|x| \leq 1} f_{h}(x, q) \leq 2(q)_{\infty}(-q)_{\infty}^{2} / \pi
$$

following (1.16) since $l(x \mid q) \leq(1+q)^{2}$ for $|x| \leq 1$.

Remark 3 We have

$$
f_{h}(x \mid 0)=2 \sqrt{1-x^{2}} / \pi, \quad \varphi_{h}(x \mid a, 0)=1 /\left(1-2 a x+a^{2}\right)
$$

for $|x|,|a|<1$.

After proper rescaling and normalization similar to the one performed in Remark 2, the case $q=1$ leads to

$$
\exp \left(-x^{2} / 2\right) / \sqrt{2 \pi}, \quad \exp \left(a x-a^{2} / 2\right)
$$


for $x, a \in \mathbb{R}$, as respectively the density of orthogonalizing measure and the generating function. For details, see [8] or [9].

\section{Main results}

Since in our approach symmetric polynomials will appear, let us introduce the following set of symmetric polynomials of $k$ variables:

$$
S_{n}^{(k)}\left(a_{1}, \ldots, a_{k} \mid q\right)=\sum_{\substack{j_{1}, \ldots, j_{k-1} \geq 0 \\ j_{1}+\cdots+j_{k-1} \leq n}}\left[j_{1}, \ldots, n-\sum_{m=1}^{k-1} j_{m}\right]_{q} a_{1}^{j_{1}} \cdots a_{k-1}^{j_{k-1}} a_{k}^{n-j_{1}-\cdots-j_{k-1}}
$$

where we denoted by $\left[j_{1}, j_{2}, \ldots, n-\sum_{m=1}^{k-1} j_{m}\right]_{q}$ the so-called $q$-multinomial coefficient defined by $\left[n_{1}, \ldots, n_{m}\right]_{q}=(q)_{n_{1}+\cdots+n_{m}} / \prod_{k=1}^{m}(q)_{n_{k}}$.

Remark 4 Notice that $S_{n}^{(k)}\left(a_{1}, \ldots, a_{k} \mid 1\right)=\left(\sum_{j=1}^{k} a_{j}\right)^{n}$.

Proof Obvious since $\left.\frac{(q)_{n}}{\prod_{m=0}^{k-1}(q)_{j m}(q)_{n-j_{1}-\cdots-j_{k-1}}}\right|_{q=1}=\frac{n !}{\left(n-\sum_{m=1}^{k-1} j_{m}\right) ! \prod_{m=1}^{k-1} j_{m} !}$.

Proposition 2 Let $q \in(-1,1)$, then:

(i)

$$
\sum_{n \geq 0} \frac{t^{n}}{(q)_{n}} S_{n}^{(k)}\left(a_{1}, \ldots, a_{k} \mid q\right)=\frac{1}{\prod_{j=1}^{k}\left(a_{i} t\right)_{\infty}}
$$

(ii) For $|t|<1$ and $\forall j=1, \ldots, k$,

$$
S_{n}^{(k)}\left(a_{1}, \ldots, a_{k} \mid q\right)=\sum_{m=0}^{n}\left[\begin{array}{c}
n \\
m
\end{array}\right]_{q} S_{m}^{(j)}\left(a_{1}, \ldots, a_{j}\right) S_{n-m}^{(k-j)}\left(a_{j+1}, \ldots, a_{k} \mid q\right) .
$$

$$
\text { If } q=1, \text { then }
$$

$$
\sum_{n \geq 0} \frac{t^{n}}{n !} S_{n}^{(k)}\left(a_{1}, \ldots, a_{k} \mid 1\right)=\exp \left(t \sum_{j=0}^{k} a_{j}\right)
$$

(iii)

$$
\left|S_{n}^{(k)}\left(a_{1}, \ldots, a_{k} \mid q\right)\right| \leq\left(\max _{1 \leq j \leq k}\left|a_{j}\right|\right)^{n} S_{n}^{(k)}(1, \ldots, 1 \mid q)
$$

Proof (i) Notice that

$$
\sum_{n \geq 0} \frac{t^{n}}{(q)_{n}} S_{n}^{(k)}\left(a_{1}, \ldots, a_{k} \mid q\right)=\sum_{n \geq 0} \sum_{\substack{j_{1}, \ldots, j_{k-1} \geq 0 \\ j_{1}+\cdots+j_{k-1} \leq n}} \frac{\left(t a_{1}\right)^{j_{1}} \cdots\left(t a_{k-1}\right)^{j_{k-1}}\left(t a_{k}\right)^{n-j_{1}-\cdots-j_{k-1}}}{\prod_{m=0}^{k-1}(q)_{j m}(q)_{n-j_{1}-\cdots-j_{k}}}
$$

Secondly recall that $\frac{1}{(a)_{\infty}}=\sum_{j \geq 0} \frac{a^{j}}{(q)_{j}}$. Now the assertion is easy. (ii) follows either from direct calculation or (i) and the properties of generating functions. (iii) We use (2.1). 
Recall (i.e., [4] or [1]) that there exist sets of orthogonal polynomials forming a part of the so-called AW scheme that are orthogonal with respect to measures with densities mentioned below. Although our main interest is in providing a simple proof of the socalled AW integral, we will list related densities for better exposition and for indicating the ways of possible generalization of AW integrals and polynomials.

So let us mention first the so-called big $q$-Hermite polynomials $\left\{h_{n}(x \mid a, q)\right\}_{n \geq-1}$ whose orthogonalizing measure has density for $|a|<1$. The density $f_{b h}$ of the orthogonalizing measure has a form (see [1, Eq. (14.18.2)]) which can be written with the help of functions $f_{h}$ and $\varphi_{h}$, namely

$$
\begin{aligned}
& f_{b h}(x \mid a, q)=f_{h}(x \mid q) \varphi_{h}(x \mid a, q) \\
& \int_{-1}^{1} h_{h}(x \mid a, q) h_{m}(x \mid a, q) f_{b h}(x \mid a, q) d x=(q)_{n} \delta_{m n} .
\end{aligned}
$$

The form of polynomials $h_{n}(x \mid a, q)$ and their relation to $q$-Hermite polynomials is not important for our considerations. It can be found, e.g., in [1, Eq. (14.26.1)] or in [10, Eqs. (2.11), (2.12)]. So, for the sake of completeness, let us remark that from (2.4) it follows immediately that for $|x| \leq 1,|a|<1$,

$$
f_{b h}(x \mid a, q)=f_{h}(x \mid q) \sum_{n \geq 0} \frac{a^{n}}{(q)_{n}} h_{n}(x \mid q)
$$

Here and below, where we will present similar expansions, convergence is almost uniform since all these expansions are in fact the Fourier series and that the Rademacher-Menshov theorem can be applied following (1.5).

Let us notice immediately that following (2.4) we have

$$
\int_{-1}^{1} h_{n}(x \mid q) f_{b h}(x \mid a, q) d x=a^{n}
$$

Secondly let us mention the so-called Al-Salam-Chihara polynomials $\left\{Q_{n}(x \mid a, b, q)\right\}_{n \geq-1}$ that are orthogonal with respect to the measure that for $|a|,|b|<1$ has the density of the form (compare [1, Eq. (14.8.2)])

$$
f_{Q}(x \mid a, b, q)=(a b)_{\infty} f_{h}(x \mid q) \varphi_{h}(x \mid a, q) \varphi_{h}(x \mid b, q) .
$$

We have the following lemma that illustrates our method and we will give a very simple proof of the well-known Poisson-Mehler formula as a corollary.

Lemma 1 For $|x| \leq 1,|a|,|b|<1$, we have

$$
f_{Q}(x \mid a, b, q)=f_{h}(x \mid q) \sum_{j=0}^{\infty} \frac{S_{j}^{(2)}(a, b)}{(q)_{j}} h_{j}(x \mid q) .
$$

Proof Following (2.6) and (1.14) we have

$$
f_{Q}(x \mid a, b, q)=(a b)_{\infty} f_{h}(x \mid q) \sum_{j, k \geq 0} \frac{a^{j} b^{k}}{(q)_{j}(q)_{k}} h_{j}(x \mid q) h_{k}(x \mid q) .
$$


Now we use (1.6) and (1.1) and change the order of summation to get

$$
\begin{aligned}
f_{Q}(x \mid a, b, q) & =(a b)_{\infty} f_{h}(x \mid q) \sum_{m \geq 0} \frac{(a b)^{m}}{(q)_{m}} \sum_{j, k \geq m} \frac{a^{j-m} b^{k-m}}{(q)_{j-m}(q)_{k-m}} h_{j-k+m-k}(x \mid q) \\
& =(a b)_{\infty} f_{h}(x \mid q) \sum_{m \geq 0} \frac{(a b)^{m}}{(q)_{m}} \sum_{n, i \geq 0} \frac{a^{n} b^{i}}{(q)_{i}(q)_{n}} h_{n+i}(x \mid q) \\
& =f_{h}(x \mid q) \sum_{s \geq 0} \frac{h_{s}(x \mid q)}{(q)_{s}} \sum_{n=0}^{s}\left[\begin{array}{l}
s \\
j
\end{array}\right]_{q} a^{n} b^{s-n} .
\end{aligned}
$$

As an immediate corollary of our result, we have

$$
\int_{-1}^{1} h_{n}(x \mid q) f_{Q}(x \mid a, b, q) d x=S_{n}^{(2)}(a, b \mid q) .
$$

Remark 5 Let $a=\rho e^{i \eta}, b=\rho e^{-i \eta}$ and denote $y=\cos \eta$. Then

(i) $S_{n}^{(2)}(a, b \mid q)=\rho^{n} h_{n}(y \mid q)$,

(ii) $v(x \mid a) v(x \mid b)=\left(1-\rho^{2}\right)^{2}-4 x y \rho\left(1+\rho^{2}\right)+4 \rho^{2}\left(x^{2}+y^{2}\right)$.

Proof (i) is an immediate consequence of (1.3). (ii) We have $v(x \mid a) v(x \mid b)=\left(1-2 \rho x e^{i \eta}+\right.$ $\left.\rho^{2} e^{2 i \eta}\right)\left(1-2 \rho x e^{-i \eta}+\rho^{2} e^{-2 i \eta}\right)$.

As a slightly more complicated corollary implied by Lemma 1, we have the following famous Poisson-Mehler (PM) expansion formula.

Corollary 1 For $|x|,|y|<1,|\rho|<1$, we have

$$
\begin{aligned}
& \frac{\left(\rho^{2}\right)_{\infty}}{\prod_{k=0}^{\infty}\left(1-\rho^{2} q^{2 k}\right)^{2}-4 x y \rho q^{k}\left(1+\rho^{2} q^{2 k}\right)+4 \rho^{2} q^{2 k}\left(x^{2}+y^{2}\right)} \\
& \quad=\sum_{j \geq 0} \frac{\rho^{j}}{(q)_{j}} h_{j}(x \mid q) h_{j}(y \mid q) .
\end{aligned}
$$

Proof We take $a=\rho e^{i \eta}, b=\rho e^{-i \eta}$ and denote $y=\cos \eta$. Now we use (2.6) and Remark 5(ii) to get the left-hand side multiplied by $f_{h}$. Then we apply Lemma 1 and Remark 5(i) to get the right-hand side of our PM formula also multiplied by $f_{h}$. Finally we cancel out $f_{h}$ which is positive on $(-1,1)$.

Remark 6 The calculations we have performed while proving Lemma 1 are very much like those performed in [4] while proving Theorem 13.1.6 concerning the Poisson kernel (or Poisson-Mehler) formula. There exist many proofs of the PM formula (see, e.g., [11] or a recently obtained very short one in [12]). In fact formula (2.9) can be dated back to Carlitz who in [13] formulated it for Rogers-Szegö polynomials. The one presented above, which seems to be one of the shortest, was obtained as a by-product and, as it has already been mentioned, is almost the same as the one presented in [4].

Notice that considering (2.8) with $a=\rho e^{i \eta}, b=\rho e^{-i \eta}$ and $y=\cos \eta$ leads in view of Remark 5(i) to

$$
\int_{-1}^{1} h_{n}(x \mid q) f_{Q}(x \mid a, b, q) d x=\rho^{n} h_{n}(y \mid q),
$$


a nice symmetric formula that appeared in [14] in a probabilistic context. Its probabilistic interpretation was exploited further in [8].

Third in our sequence of families of polynomials that constitute AW scheme is the socalled continuous dual Hahn $(\mathrm{C} 2 \mathrm{H})$ polynomials. Again their relationship to other sets of polynomials is not important. From [1, Eq. (14.3.2)] it follows that the density of measure that makes them orthogonal is given by the following formula:

$$
f_{C H}(x \mid a, b, c, q)=(a b, a c, b c)_{\infty} f_{h}(x \mid q) \varphi_{h}(x \mid a, q) \varphi_{h}(x \mid b, q) \varphi_{h}(x \mid c, q) .
$$

We have the following lemma.

\section{Lemma 2}

$$
f_{C H}(x \mid a, b, c, q)=f_{h}(x \mid q) \sum_{n \geq 0} \frac{\sigma_{n}^{(3)}(a, b, c \mid q)}{(q)_{n}} h_{n}(x \mid q),
$$

where

$$
\sigma_{n}^{(3)}(a, b, c \mid q)=\sum_{j=0}^{n}\left[\begin{array}{l}
n \\
j
\end{array}\right]_{q} q^{\left(\begin{array}{c}
j \\
2
\end{array}\right)(-a b c)^{j} S_{n-j}^{(3)}(a, b, c \mid q) .}
$$

Proof Proof is shifted to Section 4.

Remark 7 Notice that for $|t|<1$,

$$
\sum_{n \geq 0} \frac{t^{n}}{(q)_{n}} \sigma_{n}^{(3)}(a, c, b \mid q)=\frac{(a b c t)_{\infty}}{(a t, b t, c t)_{\infty}}
$$

Proof Using (2.10) we have

$$
\begin{aligned}
\sum_{n \geq 0} \frac{t^{n}}{(q)_{n}} \sigma_{n}^{(3)}(a, c, b \mid q) & =\sum_{n \geq 0} \frac{t^{n}}{(q)_{n}} \sum_{j=0}^{n}\left[\begin{array}{c}
n \\
j
\end{array}\right]_{q} q^{\left(\begin{array}{l}
j \\
2
\end{array}\right)(-a b c)^{j} S_{n-j}^{(3)}(a, b, c \mid q)} \\
& =\sum_{j=0}^{\infty} \frac{(-a b c t)^{j}}{(q)_{j}} q^{\left(\begin{array}{l}
j \\
2
\end{array}\right)} \sum_{n \geq j} \frac{t^{n-j}}{(q)_{n-j}} S_{n-j}^{(3)}(a, b, c \mid q) .
\end{aligned}
$$

Now it remains to change the index of summation in the second sum, use (2.2) and use the fact that $\sum_{j=0}^{\infty} \frac{(-a b c t)^{j}}{(q)_{j}} q^{\left(\frac{j}{2}\right)}=(a b c t)_{\infty}$.

Corollary 2 For $|a|,|b|,|c|<1$,

$$
\int_{-1}^{1} h_{n}(x \mid q) f_{C H}(x \mid a, b, c, q) d x=\sigma_{n}^{(3)}(a, b, c \mid q) .
$$

Proof Elementary.

The fourth family of polynomials that constitute AW scheme are the celebrated AskeyWilson polynomials. Again their form and relationship to other families of polynomials 
of AW scheme are not important for our considerations. Recently a relatively rich study of these relationships was done in [10], hence it may serve as the reference. We need only the form of AW density. It is given, e.g., in [1, Eq. (14.1.2)] and, after necessary adaptation to our notation, is presented below,

$$
\begin{aligned}
f_{A W}(x \mid a, b, c, d, q)= & \frac{(a b, a c, a d, b c, b d, c d)_{\infty}}{(a b c d)_{\infty}} \\
& \times f_{h}(x \mid a) \varphi_{h}(x \mid a, q) \varphi_{h}(x \mid b, q) \varphi_{h}(x \mid c, q) \varphi_{h}(x \mid d, q)
\end{aligned}
$$

for $|x| \leq 1,|a|,|b|,|c|,|d|<1$. Our main result concerns this density and is the following.

Theorem 1 For $|x| \leq 1,|a|,|b|,|c|,|d|<1$,

$$
f_{A W}(x \mid a, b, c, d, q)=f_{h}(x \mid q) \sum_{n \geq 0} \frac{\sigma_{n}^{(4)}(a, b, c, d \mid q)}{(q)_{n}} h_{n}(x \mid q),
$$

where

$$
\begin{aligned}
\sigma_{n}^{(4)}(a, b, c, d \mid q)= & \sum_{j=0}^{n}\left[\begin{array}{c}
n \\
j
\end{array}\right]_{q} \frac{(b d)_{j}}{(a b c d)_{j}} S_{n-j}^{(2)}(b, d \mid q) \\
& \times \sum_{k=0}^{j}\left[\begin{array}{l}
j \\
k
\end{array}\right]_{q}(c b)_{k} a^{k}(a d)_{j-k} c^{j-k},
\end{aligned}
$$

are symmetric functions of $a, b, c, d$.

Proof Proof is shifted to Section 4.

As immediate corollaries we have the following fact.

Corollary 3 For $\max (|a|,|b|,|c|,|d|)<1$,

$$
\int_{-1}^{1} h_{n}(x) f_{A W}(x \mid a, b, c, d, q) d x=\sigma_{n}^{(4)}(a, b, c, d \mid q)
$$

Proof Follows directly from (2.11).

Remark 8 Notice that from (2.11) in fact the value of AW integral follows since we see that $\int_{-1}^{1} f_{A W}(x|a, b, c, d| q) d x=1$, which means that the integral

$$
\begin{aligned}
& \frac{1}{2 \pi} \int_{-1}^{1} \frac{1}{\sqrt{1-x^{2}}} \prod_{n \geq 0} \frac{l\left(x \mid q^{n}\right)}{v\left(x \mid a q^{n}\right) v\left(x \mid b q^{n}\right) v\left(x \mid c q^{n}\right) v\left(x \mid d q^{n}\right)} d x \\
& \quad=\frac{(a b c d)_{\infty}}{(q, a b, a c, a d, b c, b d, c d)_{\infty}} .
\end{aligned}
$$

Equation (2.14) is nothing else but the celebrated AW integral. Notice also that recently there appeared at least two papers $[15,16]$ where $(2.14)$ was derived from much more advanced theorems. 
Remark 9 Notice also that (2.13) allows calculation of all moments of AW density. This is so since one knows the form of polynomials $h_{n}$. Moments of AW density were calculated by Corteel and Williams in 2010 in [17] using combinatorial means. For complex $a, b, c, d$ but forming conjugate pairs, this formula was also obtained independently about the same time. Namely it was done in [9] where also an elegant expansion of $\sigma_{n}^{(4)}\left(\rho_{1} e^{i \eta}, \rho_{1} e^{-i \eta}, \rho_{2} e^{i \theta}, \rho_{2} e^{-i \theta} \mid q\right)$ in terms of $h_{n}(y \mid q)$ and $h_{n}(z \mid q)$, where $\cos \eta=y$ and $\cos \theta=$ $z$, was presented.

\section{Generalization and open questions}

\subsection{Generalization}

The results presented above allow the following generalization. The cases $|q|<1$ and $q=1$ will be treated separately. First let us consider $|q|<1$.

Let us denote $\mathbf{a}^{(k)}=\left(a_{1}, \ldots, a_{k}\right), k=0,1, \ldots$. We will assume that $|x| \leq 1$ and that all parameters $a_{i}$ have absolute values less than 1 . Let us denote

$$
g_{n}\left(x \mid \mathbf{a}^{(n)}, q\right)=f_{h}(x \mid q) \prod_{j=1}^{n} \varphi_{h}\left(x \mid a_{i}, q\right)
$$

where functions $f_{h}$ and $\varphi_{h}$ were defined by (1.16) and (1.14) respectively.

We remark following (1.15) and (1.17) that

$$
g_{n}\left(x \mid \mathbf{a}^{(n)}, q\right) \leq \frac{2(q)_{\infty}(-q)_{\infty}^{2}}{\pi} \prod_{j=1}^{n} \frac{1}{\left(\left|a_{j}\right|\right)_{\infty}^{2}}
$$

for $|x| \leq 1$, and $\left|a_{i}\right|<1$ for $j=1, \ldots, n$.

We have the following general result.

Lemma 3 For every $n \geq 0$, there exist $A_{n}\left(\mathbf{a}^{(n)}, q\right)$ a symmetric function of $\mathbf{a}^{(n)}$ and a sequence of symmetric in $\mathbf{a}^{(n)}$ functions $\left\{T_{j}^{(n)}\left(\mathbf{a}^{(n)}, q\right)\right\}_{j \geq 0}$ such that for $\left|a_{k}\right|<1, k=1, \ldots, n$,

$$
g_{n}\left(x \mid \mathbf{a}^{(n)}, q\right)=A_{n}\left(\mathbf{a}^{(n)}, q\right) f_{h}(x \mid q) \sum_{j \geq 0} \frac{T_{j}^{(n)}\left(\mathbf{a}^{(n)}, q\right)}{(q)_{j}} h_{j}(x \mid q) .
$$

Moreover,

$$
\sum_{j \geq 0}\left(T_{j}^{(n)}\left(\mathbf{a}^{(n)}, q\right)\right)^{2}<\infty
$$

Proof Let $\mathcal{G}=L_{2}\left(\langle-1,1\rangle, \mathcal{F}, f_{h}\right)$ be the space of functions $h:\langle-1,1\rangle \mapsto \mathbb{R}$ such that $\int_{-1}^{1} h^{2}(x) f_{h}(x \mid q) d x$. Notice that this space is spanned by the polynomials $\left\{h_{j}(x \mid q)\right\}_{j \geq 0}$. Visibly, under our assumptions and by (1.15), $\prod_{j=1}^{n} \varphi_{h}\left(x \mid a_{i}, q\right) \in \mathcal{G}$. Now notice that $\left\{T_{j}^{(n)}\left(\mathbf{a}^{(n)}, q\right)\right\}_{j \geq 0}$ are coefficients of the Fourier expansion of the function $\prod_{j=1}^{n} \varphi_{h}\left(x \mid a_{i}, q\right)$ in $\mathcal{G}$ with respect to $\left\{h_{j}(x \mid q)\right\}_{j \geq 0}$. Since

$$
\int_{-1}^{1} f_{h}(x \mid q) \sum_{j \geq 0} \frac{T_{j}^{(n)}\left(\mathbf{a}^{(n)}, q\right)}{(q)_{j}} h_{j}(x \mid q) d x=1
$$


$A_{n}\left(\mathbf{a}^{(n)}, q\right)$ is the value of $\int_{-1}^{1} g_{n}\left(x \mid \mathbf{a}^{(n)}, q\right) d x$. Inequality (3.3) follow properties of the Fourier expansion, more precisely Perseval's identity. The fact that $A_{n}$ and $\left\{T_{j}^{(n)}\right\}_{j \geq 0}$ are symmetric follows the observations that $\prod_{j=1}^{n} \varphi_{h}\left(x \mid a_{i}, q\right)$ is symmetric.

Using formula (1.9) we can write $g_{n}$ in the following way where $h_{j}$ are $q$-Hermite polynomials defined by (1.4). Functions $A_{n}\left(\mathbf{a}^{(n)}, q\right)$ and $\left\{T_{j}^{(n)}\left(\mathbf{a}^{(n)}, q\right)\right\}_{j \geq 0}$ have the following interpretation:

$$
\int_{[-1,1]} h_{j}(x \mid q) g_{n}\left(x \mid \mathbf{a}^{(n)}, q\right) d x=A_{n}\left(\mathbf{a}^{(n)}, q\right) T_{j}^{(n)}\left(\mathbf{a}^{(n)}, q\right)
$$

for $n, j \geq 0$.

We have the following easy proposition giving recursions that are satisfied by functions $A_{n}$ and $T_{j}^{(n)}$.

Proposition 3 Let us define a new sequence of functions $\left\{H_{s}\left(\mathbf{a}^{(n)}, q\right)\right\}_{n, s \geq 0}$ of $n$ variables as follows:

$$
\sum_{m \geq 0} \frac{a_{n}^{m}}{(q)_{m}} T_{s+m}^{(n-1)}\left(\mathbf{a}^{(n-1)}, q\right)=H_{s}^{(n)}\left(\mathbf{a}^{(n)}, q\right) \sum_{m \geq 0} \frac{a_{n}^{m}}{(q)_{m}} T_{m}^{(n-1)}\left(\mathbf{a}^{(n-1)}, q\right) .
$$

Then

(i)

$$
A_{n}\left(\mathbf{a}^{(n)}, q\right)=A_{n-1}\left(\mathbf{a}^{(n-1)}, q\right) \sum_{m \geq 0} \frac{a_{n}^{m}}{(q)_{m}} T_{m}^{(n-1)}\left(\mathbf{a}^{(n-1)}, q\right),
$$

(ii)

$$
T_{j}^{(n)}\left(\mathbf{a}^{(n)}, q\right)=\sum_{s=0}^{j}\left[\begin{array}{l}
j \\
s
\end{array}\right]_{q} H_{s}^{(n-1)}\left(\mathbf{a}^{(n-1)}, q\right)\left(a_{n}\right)^{j-s}
$$

Proof Proof is shifted to Section 4.

Remark 10 The integral $\int_{-1}^{1} g_{n}\left(x \mid \mathbf{a}^{(n)}, q\right) d x$ was calculated in [18] (see also Theorem 15.3.1 in [4]) by combinatorial methods. The obtained formula is, however, very complicated. Besides, the above mentioned Theorem 15.3.1 of [4] does not provide expansion (3.2) which is automatically obtained in our approach.

Remark 11 Notice also that following Proposition 3(i) we get for $\left|a_{j}\right|<1, j=1, \ldots, 5$,

$$
\int_{-1}^{1} g_{5}\left(x \mid \mathbf{a}^{(5)}, q\right) d x=\frac{\left(\prod_{j}^{4} a_{j}\right)_{\infty}}{(q)_{\infty} \prod_{1 \leq k<m \leq 4}\left(a_{k} a_{m}\right)_{\infty}} \sum_{j \geq 0} \frac{a_{5}^{j}}{(q)_{j}} \sigma_{j}^{(4)}\left(a_{1}, a_{2}, a_{3}, a_{4} \mid q\right) .
$$

For $q=0$ the calculations presented in (3.4) can be carried out completely and the concise form can be obtained. This is possible due to the following simplified form of (2.12).

Theorem 2 Under $\left|a_{j}\right|<1, j=1, \ldots, 5$, we have 
(i)

$$
\begin{aligned}
& \sigma_{n}^{(4)}\left(a_{1}, a_{2}, a_{3}, a_{4} \mid 0\right) \\
& =S_{n}^{(2)}\left(a_{2}, a_{4} \mid 0\right)+\frac{\left(1-a_{2} d\right)\left(1-a_{1} a_{4}\right)}{\left(1-a_{1} a_{2} a_{3} a_{4}\right)} a_{3} S_{n-1}^{(3)}\left(a_{2}, a_{3}, a_{4} \mid 0\right) \\
& \quad+\frac{\left(1-a_{2} a_{4}\right)\left(1-a_{3} a_{2}\right)}{\left(1-a_{1} a_{2} a_{3} a_{4}\right)} a_{1} S_{n-1}^{(3)}\left(a_{1}, a_{2}, a_{4} \mid 0\right) \\
& +\frac{\left(1-a_{2} a_{4}\right)\left(1-a_{2} a_{3}\right)\left(1-a_{1} a_{4}\right) a_{1} a_{3}}{\left(1-a_{1} a_{2} a_{3} a_{4}\right)} S_{n-2}^{(4)}\left(a_{1}, a_{2}, a_{3}, a_{4} \mid 0\right)
\end{aligned}
$$

(ii)

$$
\int_{-1}^{1} g_{5}\left(x \mid \mathbf{a}^{(5)}, 0\right) d x=\frac{1-\chi_{4}\left(\mathbf{a}^{(5)}\right)+\chi_{5}\left(\mathbf{a}^{(5)}\right) \chi_{1}\left(\mathbf{a}^{(5)}\right)-\chi_{5}^{2}\left(\mathbf{a}^{(5)}\right)}{\prod_{1 \leq j<k \leq 5}\left(1-a_{j} a_{k}\right)},
$$

where $\chi_{1}, \ldots, \chi_{5}$ denote respectively first five elementary symmetric functions of the vector $\mathbf{a}^{(5)}$. That is, $\chi_{j}\left(\mathbf{a}^{(k)}\right)=\sum_{1 \leq n_{1}<n_{2} \cdots<n_{j} \leq k} \prod_{m=1}^{j} a_{n_{m}}$.

Proof Proof is shifted to Section 4.

For $q=1$, the problem of finding sequences $A_{n}\left(\mathbf{a}^{(n)} \mid 1\right)$ and $\left\{T_{j}^{(n)}\left(\mathbf{a}^{(n)}, 1\right)\right\}_{j \geq 0}$ can be solved completely and trivially. Namely we have the following.

\section{Proposition 4}

$$
\begin{aligned}
& A_{n}\left(\mathbf{a}^{(n)} \mid 1\right)=\exp \left(\sum_{1 \leq j<k \leq n} a_{j} a_{k}\right), \\
& T_{j}^{(n)}\left(\mathbf{a}^{(n)}, 1\right)=\left(\sum_{k=1}^{n} a_{k}\right)^{j} .
\end{aligned}
$$

Proof Using Remark 3 we get

$$
\begin{aligned}
g_{n}\left(x \mid \mathbf{a}^{(n)}, 1\right)= & \exp \left(-x^{2} / 2+x \sum_{j=1}^{n} a_{j}-\frac{1}{2} \sum_{j=1}^{n} a_{j}^{2}\right) / \sqrt{2 \pi} \\
= & \frac{1}{\sqrt{2 \pi}} \exp \left(\frac{1}{2}\left(\left(\sum_{j=1}^{n} a_{j}\right)^{2}-\sum_{j=1}^{n} a_{j}^{2}\right)\right) \\
& \times \exp \left(-x^{2} / 2+x \sum_{j=1}^{n} a_{j}-\frac{1}{2}\left(\sum_{j=1}^{n} a_{j}\right)^{2}\right) \\
= & \exp \left(\sum_{1 \leq j<k \leq n} a_{j} a_{k}\right) \frac{\exp \left(-x^{2} / 2\right)}{\sqrt{2 \pi}} \sum_{j \geq 0} \frac{\left(\sum_{k=1}^{n} a_{k}\right)^{j}}{j !} H_{j}(x) .
\end{aligned}
$$

\subsection{Unsolved problems and open questions}

\subsubsection{Questions}

- What are the compact forms of functions $\left\{T_{j}^{(n)}\left(\mathbf{a}^{(n)}, q\right)\right\}_{j \geq 0, n \geq 5}$ and $\left\{A_{n}\left(\mathbf{a}^{(n)}, q\right)\right\}_{n \geq 5}$ ? 
- What are the compact forms of these functions for $q=0$ (free probability case)?

- Following formula for $\int_{-1}^{1} g_{5}\left(x \mid \mathbf{a}^{(5)}, 0\right) d x$ given in assertion (ii) of Theorem 2 is it true that

$$
\int_{-1}^{1} g_{5}\left(x \mid \mathbf{a}^{(5)}, q\right) d x=\frac{\left(\chi_{4}\left(\mathbf{a}^{(5)}\right)-\chi_{5}\left(\mathbf{a}^{(5)}\right) \chi_{1}\left(\mathbf{a}^{(5)}\right)+\chi_{5}^{2}\left(\mathbf{a}^{(5)}\right)\right)_{\infty}}{\prod_{1 \leq j<k \leq 5}\left(a_{j} a_{k}\right)_{\infty}} ?
$$

Notice that for $a_{5}=0$ it would reduce to AW integral.

- It would be valuable to get values $\left\{A_{n}\left(\mathbf{a}^{(n)}, q\right)\right\}$ for $n=8,12$ and so on for complex values of parameters $\mathbf{a}^{(n)}$ but forming conjugate pairs. It would be also fascinating to find polynomials that would be orthogonalized by densities obtained in this way.

This problem follows the probabilistic interpretation of Askey-Wilson density rescaled with complex parameters. Such an interpretation of finite Markov chains of length at least 3 was presented in $[9,10]$. Let $\left\{X_{1}, X_{2}, X_{3}\right\}$ denote this finite Markov chain. Recall that then AW density can be interpreted as the conditional density of $X_{2} \mid X_{1}, X_{3}$.

It would be exciting to find out if, for say $n=8$, a similar probabilistic interpretation could be established. That is, if we could define five-dimensional random vector $\left(X_{1}, \ldots, X_{5}\right)$ with normalized function $g_{8}\left(x \mid \mathbf{a}^{(8)}, q\right)$ as the conditional density $X_{3} \mid X_{1}, X_{2}$, $X_{4}, X_{5}$. Note that then the chain $\left(X_{1}, \ldots, X_{5}\right)$ could not be Markov.

Similar questions apply to the case $n=12,16, \ldots$.

\subsubsection{Unsolved related problems and direction of further research}

1. In [2] we find Theorem 10.8.2 which is due to Gasper and Rahman (1990) and which can be stated in our notation. For $\max _{1 \leq j \leq 5}\left|a_{j}\right|<1,|q|<1$, we have

$$
\int_{-1}^{1} \frac{g_{5}\left(x \mid \mathbf{a}^{(5)}, q\right)}{\varphi_{h}\left(x \mid \prod_{j=1}^{5} a_{j}, q\right)} d x=\frac{\prod_{j=1}^{5}\left(\prod_{k=1, k \neq j}^{5} a_{k}\right)_{\infty}}{\prod_{1 \leq j<k \leq 5}\left(a_{j} a_{k}\right)_{\infty}} .
$$

This result suggests considering the following functions:

$$
G_{n, m}\left(x \mid \mathbf{a}^{(n)}, \mathbf{b}^{(m)}, q\right)=f_{h}(x \mid q) \frac{\prod_{j=1}^{n} \varphi_{h}\left(x \mid a_{i}, q\right)}{\prod_{k=1}^{m} \varphi_{h}\left(x \mid b_{k}, q\right)},
$$

where $\mathbf{a}^{(n)}$ and $\mathbf{b}^{(m)}$ are certain vectors of dimensions respectively $n$ and $m$, find its integrals over $[-1,1]$ and expansions similar to (3.2).

2. Recently a paper [19] on $q$-Laplace transform, where many analogies to ordinary case were indicated, has appeared. What would a $q$-Laplace transform of the distributions that were considered above be?

\section{Proofs}

Proof of Lemma 2 We have

$$
\begin{aligned}
& \sum_{k, n, m \geq 0} \frac{a^{n}}{(q)_{n}} \frac{b^{m}}{(q)_{m}} \frac{c^{k}}{(q)_{k}} h_{n}(x \mid q) h_{m}(x \mid q) h_{k}(x \mid q) \\
& \quad=\frac{1}{(a b)_{\infty}} \sum_{j \geq 0} \frac{h_{j}(x \mid q)}{(q)_{j}} \sum_{m=0}^{\infty} \frac{c^{m}}{(q)_{m}} \sum_{k=0}^{j}\left[\begin{array}{l}
j \\
k
\end{array}\right]_{q} c^{k} S_{m+j-k}^{(2)}(a, b \mid q) .
\end{aligned}
$$


Since obviously $S_{n}^{(2)}(a, b \mid q)=a^{n} w_{n}(b / a \mid q)$, we get

$$
\begin{aligned}
& \sum_{k, n, m \geq 0} \frac{a^{n}}{(q)_{n}} \frac{b^{m}}{(q)_{m}} \frac{c^{k}}{(q)_{k}} h_{n}(x \mid q) h_{m}(x \mid q) h_{k}(x \mid q) \\
& \quad=\frac{1}{(a b)_{\infty}} \sum_{j \geq 0} \frac{h_{j}(x \mid q)}{(q)_{j}} \sum_{m=0}^{\infty} \frac{c^{m}}{(q)_{m}} \sum_{k=0}^{j}\left[\begin{array}{l}
j \\
k
\end{array}\right]_{q} c^{k} a^{m+j-k} w_{m+j-k}(b / a \mid q) \\
& =\frac{1}{(a b)_{\infty}} \sum_{j \geq 0} \frac{h_{j}(x \mid q)}{(q)_{j}} \sum_{k=0}^{j}\left[\begin{array}{l}
j \\
k
\end{array}\right]_{q} c^{k} a^{j-k} \sum_{m=0}^{\infty} \frac{(a c)^{m}}{(q)_{m}} w_{m+j-k}(b / a \mid q) .
\end{aligned}
$$

Now we apply formula (1.7) and get

$$
\begin{aligned}
& \sum_{k, n, m \geq 0} \frac{a^{n}}{(q)_{n}} \frac{b^{m}}{(q)_{m}} \frac{c^{k}}{(q)_{k}} h_{n}(x \mid q) h_{m}(x \mid q) h_{k}(x \mid q) \\
& =\frac{1}{(a b, b c, a c)_{\infty}} \sum_{j \geq 0} \frac{h_{j}(x \mid q)}{(q)_{j}} \sum_{k=0}^{j}\left[\begin{array}{l}
j \\
k
\end{array}\right]_{q} c^{k} a^{j-k} \mu_{j-k}(b / a \mid a c, q) \frac{1}{(b c)_{\infty}(b c)_{\infty}} \\
& =\frac{1}{(a b, b c, a c)_{\infty}} \sum_{j \geq 0} \frac{h_{j}(x \mid q)}{(q)_{j}} \sum_{l=0}^{j}\left[\begin{array}{l}
n \\
l
\end{array}\right]_{q} c^{n-l} a^{l}\left(\frac{b}{a}\right)^{j}\left(\frac{a}{b}\right)^{j} \mu_{j}\left(\left(\frac{a}{b}\right)^{-1} \mid a c, q\right) .
\end{aligned}
$$

Now we use (1.12) and Proposition 2(ii) and get

$$
\begin{aligned}
& \sum_{k, n, m \geq 0} \frac{a^{n}}{(q)_{n}} \frac{b^{m}}{(q)_{m}} \frac{c^{k}}{(q)_{k}} h_{n}(x \mid q) h_{m}(x \mid q) h_{k}(x \mid q) \\
& =\frac{1}{(a b, b c, a c)_{\infty}} \sum_{j \geq 0} \frac{h_{j}(x \mid q)}{(q)_{j}} \sum_{l=0}^{j}\left[\begin{array}{l}
j \\
l
\end{array}\right]_{q} c^{j-l} b^{l} \sum_{k=0}^{l}\left[\begin{array}{l}
l \\
k
\end{array}\right]_{q}(-a c)^{k} q^{\left(\begin{array}{c}
k \\
2
\end{array}\right)} w_{l-k}\left(\frac{a}{b} \mid q\right) \\
& =\frac{1}{(a b, b c, a c)_{\infty}} \sum_{j \geq 0} \frac{h_{j}(x \mid q)}{(q)_{j}} \sum_{k=0}^{j}\left[\begin{array}{l}
j \\
k
\end{array}\right]_{q}(-a c)^{k} q^{\left(\begin{array}{l}
k \\
2
\end{array}\right)} \sum_{l=k}^{j}\left[\begin{array}{l}
j-k \\
l-k
\end{array}\right]_{q} c^{j-l} b^{l} w_{l-k}\left(\frac{a}{b} \mid q\right) \\
& =\frac{1}{(a b, b c, a c)_{\infty}} \sum_{j \geq 0} \frac{h_{j}(x \mid q)}{(q)_{j}} \sum_{k=0}^{j}\left[\begin{array}{l}
j \\
k
\end{array}\right]_{q}(-a c)^{k} q^{\left(\begin{array}{c}
k \\
2
\end{array}\right)} \sum_{m=0}^{j-k}\left[\begin{array}{c}
j-k \\
m
\end{array}\right]_{q} c^{j-k-m} b^{k+m} w_{m}(a / b \mid q) \\
& =\frac{1}{(a b, b c, a c)_{\infty}} \sum_{j \geq 0} \frac{h_{j}(x \mid q)}{(q)_{j}} \sum_{k=0}^{j}\left[\begin{array}{l}
j \\
k
\end{array}\right]_{q}(-a b c)^{k} q^{\left(\begin{array}{c}
k \\
2
\end{array}\right)} \sum_{m=0}^{j-k}\left[\begin{array}{c}
j-k \\
m
\end{array}\right]_{q} c^{j-k-m} S_{m}^{(2)}(a, b \mid q) .
\end{aligned}
$$

Proof of Theorem 1 Applying (2.7) we get

$$
\begin{aligned}
& \sum_{k, n, m, j \geq 0} \frac{a^{n}}{(q)_{n}} \frac{b^{m}}{(q)_{m}} \frac{c^{k}}{(q)_{k}} \frac{d^{j}}{(q)_{j}} h_{n}(x \mid q) h_{m}(x \mid q) h_{k}(x \mid q) h_{j}(x \mid q) \\
& =\frac{1}{(a b, c d)_{\infty}} \sum_{m, k \geq 0} \frac{S_{m}^{(2)}(a, b) S_{k}^{(2)}(c, d)}{(q)_{m}(q)_{k}} h_{m}(x \mid q) h_{k}(x \mid q) \\
& =\frac{1}{(a b, c d)_{\infty}} \sum_{m, k \geq 0} \frac{S_{m}^{(2)}(a, b) S_{k}^{(2)}(c, d)}{(q)_{m}(q)_{k}}
\end{aligned}
$$




$$
\begin{aligned}
& \times \sum_{j=0}^{\min (m, k)}\left[\begin{array}{c}
m \\
j
\end{array}\right]_{q}\left[\begin{array}{l}
k \\
j
\end{array}\right]_{q}(q)_{j} h_{m+k-2 j}(x \mid q) \\
= & \frac{1}{(a b, c d)_{\infty}} \sum_{j \geq 0} \frac{(a c)^{j}}{(q)_{j}} \sum_{m, k \geq j} \frac{a^{m-j} c^{k-j} w_{m}(b / a \mid q) w_{k}(d / c \mid q)}{(q)_{m-j}(q)_{k-j}} h_{m-j+k-j}(x \mid q),
\end{aligned}
$$

and further

$$
\begin{aligned}
& \sum_{k, n, m, j \geq 0} \frac{a^{n}}{(q)_{n}} \frac{b^{m}}{(q)_{m}} \frac{c^{k}}{(q)_{k}} \frac{d^{j}}{(q)_{j}} h_{n}(x \mid q) h_{m}(x \mid q) h_{k}(x \mid q) h_{j}(x \mid q) \\
& =\frac{1}{(a b, c d)_{\infty}} \sum_{j \geq 0} \frac{(a c)^{j}}{(q)_{j}} \sum_{s, t \geq 0} \frac{a^{s} c^{t} w_{s+j}(b / a \mid q) w_{t+j}(d / c \mid q)}{(q)_{s}(q)_{t}} h_{s+t}(x \mid q) \\
& =\frac{1}{(a b, c d)_{\infty}} \sum_{j \geq 0} \frac{(a c)^{j}}{(q)_{j}} \sum_{n \geq 0} \frac{h_{n}(x \mid q)}{(q)_{n}} \\
& \quad \times \sum_{k=0}^{n}\left[\begin{array}{l}
n \\
k
\end{array}\right]_{q} a^{k} c^{n-k} w_{k+j}(b / a \mid q) w_{j+n-k}(d / c \mid q) \\
& =\frac{1}{(a b, c d)_{\infty}} \sum_{n \geq 0} \frac{h_{n}(x \mid q)}{(q)_{n}} \sum_{k=0}^{n}\left[\begin{array}{l}
n \\
k
\end{array}\right]_{q} a^{k} c^{n-k} \\
& \quad \times \sum_{j \geq 0} \frac{(a c)^{j}}{(q)_{j}} w_{k+j}(b / a \mid q) w_{j+n-k}(d / c \mid q) .
\end{aligned}
$$

Now we apply Carlitz formulae (1.10) and (1.11) to get

$$
\begin{aligned}
\sum_{k, n, m, j \geq 0} \frac{a^{n}}{(q)_{n}} \frac{b^{m}}{(q)_{m}} \frac{c^{k}}{(q)_{k}} \frac{d^{j}}{(q)_{j}} h_{n}(x \mid q) h_{m}(x \mid q) h_{k}(x \mid q) h_{j}(x \mid q) \\
=\frac{(a b c d)_{\infty}}{(a b, c d, a c, b c, a d, b d)_{\infty}} \sum_{n \geq 0} \frac{h_{n}(x \mid q)}{(q)_{n}} \sum_{k=0}^{n}\left[\begin{array}{l}
n \\
k
\end{array}\right]_{q} a^{k} c^{n-k} \\
\quad \times \sum_{s=0}^{k} \sum_{t=0}^{n-k}\left[\begin{array}{l}
k \\
s
\end{array}\right]_{q}\left[\begin{array}{c}
n-k \\
t
\end{array}\right]_{q} \frac{(c b)_{s}(a d)_{t}(b d)_{s+t}}{(a b c d)_{s+t}}\left(\frac{b}{a}\right)^{k-s}\left(\frac{d}{c}\right)^{n-k-t} \\
=\frac{(a b c d)_{\infty}}{(a b, c d, a c, b c, a d, b d)_{\infty}} \sum_{n \geq 0} \frac{h_{n}(x \mid q)}{(q)_{n}} \sum_{k=0}^{n}\left[\begin{array}{l}
n \\
k
\end{array}\right]_{q} \\
\quad \times \sum_{s=0}^{k} \sum_{t=0}^{n-k}\left[\begin{array}{l}
k \\
s
\end{array}\right]_{q}\left[\begin{array}{c}
n-k \\
t
\end{array}\right]_{q} \frac{(c b)_{s}(a d)_{t}(b d)_{s+t}}{(a b c d)_{s+t}} a^{s} b^{k-s} c^{t} d^{n-k-t} .
\end{aligned}
$$

Thus it remains to show that for every $n \geq 0$,

$$
\begin{gathered}
\sum_{k=0}^{n}\left[\begin{array}{l}
n \\
k
\end{array}\right]_{q} \sum_{s=0}^{k} \sum_{t=0}^{n-k}\left[\begin{array}{l}
k \\
s
\end{array}\right]_{q}\left[\begin{array}{c}
n-k \\
t
\end{array}\right]_{q} \frac{(c b)_{s}(a d)_{t}(b d)_{s+t}}{(a b c d)_{s+t}} a^{s} b^{k-s} c^{t} d^{n-k-t} \\
=\sum_{j=0}^{n}\left[\begin{array}{l}
n \\
j
\end{array}\right]_{q} \frac{(b d)_{j}}{(a b c d)_{j}} S_{n-j}^{(2)}(b, d \mid q) \sum_{k=0}^{j}\left[\begin{array}{l}
j \\
k
\end{array}\right]_{q}(c b)_{k} a^{k}(a d)_{j-k} c^{j-k} .
\end{gathered}
$$


This fact is a result of the following calculations:

$$
\begin{aligned}
\sum_{k=0}^{n}\left[\begin{array}{l}
n \\
k
\end{array}\right]_{q} \sum_{s=0}^{k} \sum_{t=0}^{n-k}\left[\begin{array}{l}
k \\
s
\end{array}\right]_{q}\left[\begin{array}{c}
n-k \\
t
\end{array}\right]_{q} \frac{(c b)_{s}(a d)_{t}(b d)_{s+t}}{(a b c d)_{s+t}} a^{s} b^{k-s} c^{t} d^{n-k-t} \\
=\sum_{s, t \geq 0, s+t \leq n} \frac{(q)_{n}}{(q)_{s}(q)_{t}(q)_{n-s-t}} \frac{(c b)_{s}(a d)_{t}(b d)_{s+t}}{(a b c d)_{s+t}} a^{s} c^{t} \sum_{k=s \vee n-t}^{n}\left[\begin{array}{c}
n-t-s \\
k-s
\end{array}\right]_{q} b^{k-s} d^{n-k-t} \\
=\sum_{s, t \geq 0, s+t \leq n} \frac{(q)_{n}}{(q)_{s}(q)_{t}(q)_{n-s-t}} \frac{(c b)_{s}(a d)_{t}(b d)_{s+t}}{(a b c d)_{s+t}} a^{s} c^{t} \\
\quad \times \sum_{m=0 \vee n-t-s}^{n-s}\left[\begin{array}{c}
n-t-s \\
m
\end{array}\right]_{q} b^{m} d^{n-s-m-t} \\
=\sum_{s, t \geq 0, s+t \leq n} \frac{(q)_{n}}{(q)_{s}(q)_{t}(q)_{n-s-t}} \frac{(c b)_{s}(a d)_{t}(b d)_{s+t}}{(a b c d)_{s+t}} a^{s} c^{t} S_{n-t-s}^{(2)}(b, d \mid q) .
\end{aligned}
$$

Now we introduce new indices of summation $j=t+s, k=s$. We have then

$$
\begin{aligned}
& \sum_{s, t \geq 0, s+t \leq n} \frac{(q)_{n}}{(q)_{s}(q)_{t}(q)_{n-s-t}} \frac{(c b)_{s}(a d)_{t}(b d)_{s+t}}{(a b c d)_{s+t}} a^{s} c^{t} S_{n-t-s}^{(2)}(b, d \mid q) \\
& =\sum_{j=0}^{n}\left[\begin{array}{l}
n \\
j
\end{array}\right]_{q} \frac{(b d)_{j}}{(a b c d)_{j}} S_{n-j}^{(2)}(b, d \mid q) \sum_{k=0}^{j}\left[\begin{array}{l}
j \\
k
\end{array}\right]_{q}(c b)_{k} a^{k}(a d)_{j-k} c^{j-k} .
\end{aligned}
$$

Proof of Proposition 3 Notice that for $n=0$ our formulae are true since we have $g_{1}\left(x \mid a_{1}, q\right)=$ $f_{h}(x \mid q) \varphi_{h}\left(x \mid a_{1}, q\right)=f_{h}(x \mid q) \sum_{m \geq 0} \frac{a_{1}^{m}}{(q)_{m}} h_{m}(x \mid q)$. So $T_{m}^{(1)}\left(a_{1}, q\right)=a_{1}^{m}$ and $A_{1}\left(a_{1}, q\right)=1$. Next notice that

$$
g_{n+1}\left(x \mid \mathbf{a}^{(n+1)}, q\right)=g_{n}\left(x \mid \mathbf{a}^{(n)}, q\right) \varphi_{h}\left(x \mid a_{n+1}, q\right)
$$

where we understand $\mathbf{a}^{(n+1)}=\left(a_{1}, \ldots, a_{n}, a_{n+1}\right)$. So by induction assumption the left-hand side of (3.2) is equal to

$$
A_{n+1}\left(\mathbf{a}^{(n+1)}, q\right) f_{h}(x \mid q) \sum_{j \geq 0} \frac{T_{j}^{(n+1)}\left(\mathbf{a}^{(n+1)}, q\right)}{(q)_{j}} h_{j}(x \mid q),
$$

while the right-hand side to

$$
A_{n}\left(\mathbf{a}^{(n)}, q\right) f_{h}(x \mid q) \sum_{j, k \geq 0} \frac{T_{j}^{(n)}\left(\mathbf{a}^{(n)}, q\right) a_{n+1}^{k}}{(q)_{j}(q)_{k}} h_{j}(x \mid q) h_{k}(x \mid q) .
$$

We apply again (1.6) to get

$$
\begin{aligned}
& \sum_{j, k \geq 0} \frac{T_{j}^{(n)}\left(\mathbf{a}^{(n)}, q\right) a_{n+1}^{k}}{(q)_{j}(q)_{k}} h_{j}(x \mid q) h_{k}(x \mid q) \\
& \quad=\sum_{j, k \geq 0} \frac{T_{j}^{(n)}\left(\mathbf{a}^{(n)}, q\right) a_{n+1}^{k}}{(q)_{j}(q)_{k}} \sum_{m=0}^{j \wedge k}\left[\begin{array}{c}
k \\
m
\end{array}\right]_{q}\left[\begin{array}{l}
j \\
m
\end{array}\right]_{q}(q)_{m} h_{j+k-2 m}(x \mid q)
\end{aligned}
$$




$$
\begin{aligned}
& =\sum_{m \geq 0} \frac{a_{n+1}^{m}}{(q)_{m}} \sum_{k, j \geq m} \frac{a_{n+1}^{k-m} T_{j}^{(n)}\left(\mathbf{a}^{(n)}, q\right)}{(q)_{k-m}(q)_{j-m}} h_{j+k-2 m}(x \mid q) \\
& =\sum_{m \geq 0} \frac{a_{n+1}^{m}}{(q)_{m}} \sum_{s, t \geq 0} \frac{a_{n+1}^{s} T_{t+m}^{(n)}\left(\mathbf{a}^{(n)}, q\right)}{(q)_{s}(q)_{t}} h_{s+t}(x \mid q) .
\end{aligned}
$$

Next we introduce new indices of summation $r=s+t$ and $j=s$ and get

$$
\begin{aligned}
& \sum_{j, k \geq 0} \frac{T_{j}^{(n)}\left(\mathbf{a}^{(n)}, q\right) a_{n+1}^{k}}{(q)_{j}(q)_{k}} h_{j}(x \mid q) h_{k}(x \mid q) \\
& =\sum_{m \geq 0} \frac{a_{n+1}^{m}}{(q)_{m}} \sum_{r=0}^{\infty} \frac{h_{r}(x \mid q)}{(q)_{r}} \sum_{j=0}^{r}\left[\begin{array}{l}
r \\
j
\end{array}\right]_{q} a_{n+1}^{j} T_{m+r-j}^{(n)}\left(\mathbf{a}^{(n)}, q\right) \\
& =\sum_{r=0}^{\infty} \frac{h_{r}(x \mid q)}{(q)_{r}} \sum_{j=0}^{r}\left[\begin{array}{l}
r \\
j
\end{array}\right]_{q} a_{n+1}^{j} \sum_{m \geq 0} \frac{a_{n+1}^{m}}{(q)_{m}} T_{m+r-j}^{(n)}\left(\mathbf{a}^{(n)}, q\right) \\
& =\sum_{m \geq 0} \frac{a_{n+1}^{m}}{(q)_{m}} T_{m}^{(n)}\left(\mathbf{a}^{(n)}, q\right) \sum_{r=0}^{\infty} \frac{h_{r}(x \mid q)}{(q)_{r}} \sum_{j=0}^{r}\left[\begin{array}{l}
r \\
j
\end{array}\right]_{q} a_{n+1}^{j} H_{r-j}^{(n)}\left(\mathbf{a}^{(n)}, q\right) \\
& =\frac{A_{n+1}\left(\mathbf{a}^{(n+1)}, q\right)}{A_{n}\left(\mathbf{a}^{(n)}, q\right)} \sum_{r=0}^{\infty} \frac{h_{r}(x \mid q)}{(q)_{r}} \sum_{j=0}^{r}\left[\begin{array}{l}
r \\
j
\end{array}\right]_{q} a_{n+1}^{j} H_{r-j}^{(n)}\left(\mathbf{a}^{(n)}, q\right) .
\end{aligned}
$$

Proof of Theorem 2 We use (2.12) and utilizing Remark 1 we get

$$
\begin{aligned}
\sigma_{n}^{(4)}(a, b, c, d \mid 0)= & S_{n}^{(2)}(b, d \mid 0)+\frac{(1-b d)}{(1-a b c d)} \sum_{j=1}^{n} S_{n-j}^{(2)}(b, d \mid q) \\
& \times\left((1-a d) c^{j}+(1-c b) a^{j}+(1-c b)(1-a d) a c \sum_{k=1}^{j-1} a^{k-1} c^{j-1-k}\right) .
\end{aligned}
$$

And further

$$
\begin{aligned}
\sigma_{n}^{(4)}(a, b, c, d \mid 0)= & S_{n}^{(2)}(b, d \mid 0)+\frac{(1-b d)}{(1-a b c d)} \sum_{j=1}^{n} S_{n-j}^{(2)}(b, d \mid q)\left((1-a d) c^{j}+(1-c b) a^{j}\right. \\
& \left.+(1-c b)(1-a d) a c S_{j-2}^{(2)}(a, c \mid 0)\right) \\
= & S_{n}^{(2)}(b, d \mid 0)+\frac{(1-b d)}{(1-a b c d)} \sum_{j=1}^{n} S_{n-j}^{(2)}(b, d \mid q)\left((1-a d) c^{j}+(1-c b) a^{j}\right) \\
& +\frac{(1-b d)(1-c b)(1-a d) a c}{(1-a b c d)} \sum_{j=2}^{n} S_{n-j}^{(2)}(b, d \mid q) S_{j-2}^{(2)}(a, c \mid 0) .
\end{aligned}
$$

Now we use formula (2.3). Then we replace $a$ by $a_{1}, b$ by $a_{2}$ and so on. Finally we use formulae (3.4) and (2.2) remembering that $(0)_{n}=1$ leads to our integral formula. 


\section{Acknowledgements}

The author is very grateful to an unknown referee for pointing out additional references and just evaluation of the paper.

\section{Received: 25 August 2014 Accepted: 26 November 2014 Published: 15 Dec 2014}

\section{References}

1. Koekoek, R, Lesky, PA, Swarttouw, RF: Hypergeometric Orthogonal Polynomials and Their q-Analogues. Springer Monographs in Mathematics. Springer, Berlin (2010). ISBN:978-3-642-05013-8 With a foreword by Tom H. Koornwinder. MR2656096 (2011e:33029)

2. Andrews, GE, Askey, R, Roy, R: Special Functions. Encyclopedia of Mathematics and Its Applications, vol. 71. Cambridge University Press, Cambridge (1999). ISBN:0-521-62321-9; ISBN:0-521-78988-5. MR1688958 (2000g:33001) (2002k:33011)

3. Szabłowski, PJ: On peculiar properties of generating functions of some orthogonal polynomials. J. Phys. A, Math. Theor. 45, 365207 (2012). arXiv:1204.0972

4. Ismail, MEH: Classical and Quantum Orthogonal Polynomials in One Variable. Encyclopedia of Mathematics and Its Applications, vol. 98. Cambridge University Press, Cambridge (2005). ISBN:0-521-78201-5. MR2191786 (2007f:33001). With two chapters by Walter Van Assche. With a foreword by Richard A. Askey

5. Szabłowski, PJ: On the $q$-Hermite polynomials and their relationship with some other families of orthogonal polynomials. Demonstr. Math. 66(4), 679-708 (2013). arXiv:1101.2875

6. Carlitz, L: Some polynomials related to theta functions. Ann. Mat. Pura Appl. (4) 41, 359-373 (1956). MR0078510 $(17,1205 e)$

7. Carlitz, L: Generating functions for certain Q-orthogonal polynomials. Collect. Math. 23, 91-104 (1972). MR0316773 (47 \#5321)

8. Bryc, W, Matysiak, W, Szabłowski, PJ: Probabilistic aspects of Al-Salam-Chihara polynomials. Proc. Am. Math. Soc. 133(4), 1127-1134 (2005) (electronic). MR2117214 (2005m:33033)

9. Szabłowski, PJ: On the structure and probabilistic interpretation of Askey-Wilson densities and polynomials with complex parameters. J. Funct. Anal. 261(3), 635-659 (2011). arXiv:1011.1541. MR2799574

10. Szabłowski, PJ: Befriending Askey-Wilson polynomials. Infin. Dimens. Anal. Quantum Probab. Relat. Top. 17(3), 1450015 (2014). arXiv:1111.0601

11. Bressoud, DM: A simple proof of Mehler's formula for q-Hermite polynomials. Indiana Univ. Math. J. 29(4), 577-580 (1980). MR0578207 (81f:33009)

12. Szabłowski, PJ: Expansions of one density via polynomials orthogonal with respect to the other. J. Math. Anal. Appl. 383(1), 35-54 (2011). arXiv:1011.1492. MR2812716

13. Carlitz, L: Some polynomials related to theta functions. Duke Math. J. 24, 521-527 (1957). MR0090672 (19,849e)

14. Bryc, W: Stationary random fields with linear regressions. Ann. Probab. 29(1), 504-519 (2001). MR1825162 (2002d:60014)

15. Liu, Z-G: An identity of Andrews and the Askey-Wilson integral. Ramanujan J. 19(1), 115-119 (2009). MR2501242 (2010g:33007)

16. Ma, XR: A new proof of the Askey-Wilson integral via a five-variable Ramanujan's reciprocity theorem. Ramanujan J. 24(1), 61-65 (2011). MR2765601

17. Corteel, S, Williams, LK: Staircase tableaux, the asymmetric exclusion process, and Askey-Wilson polynomials. Proc Natl. Acad. Sci. USA 107(15), 6726-6730 (2010). MR2630104

18. Ismail, MEH, Stanton, D, Viennot, G: The combinatorics of $q$-Hermite polynomials and the Askey-Wilson integral. Eur. J. Comb. 8(4), 379-392 (1987). MR0930175 (89h:33015)

19. Chung, WS, Kim, T, Kwon, HI: On the $q$-analog of the Laplace transform. Russ. J. Math. Phys. 21(2), 156-168 (2014). MR3215667

10.1186/1687-1847-2014-316

Cite this article as: Szabłowski: Askey-Wilson integral and its generalizations. Advances in Difference Equations 2014, 2014:316

\section{Submit your manuscript to a SpringerOpen ${ }^{\circ}$ journal and benefit from:}

- Convenient online submission

- Rigorous peer review

- Immediate publication on acceptance

- Open access: articles freely available online

- High visibility within the field

- Retaining the copyright to your article 